\title{
Development and Validation of an Ecofriendly HPLC-UV Method for Determination of Capecitabine in Human Plasma: Application to Pharmacokinetic Studies
}

\author{
Sepideh Hassanlou ${ }^{a}$, Mehdi Rajabi ${ }^{a}$, Abdol ali Shahrasbi $^{\mathrm{b}}$ and Minoo Afshar, ${ }^{\mathrm{c}, *}$ \\ ${ }^{a}$ Department of Clinical Pharmacy, Faculty of Pharmacy, Pharmaceutical Sciences Branch, Islamic Azad University (IAUPS), \\ Tehran 193956466, Iran. \\ ${ }^{b}$ Booali Hospital, Tehran Medical Unit, Islamic Azad University, Tehran, Iran. \\ ${ }^{c}$ Department of Pharmaceutics, Faculty of Pharmacy, Pharmaceutical Sciences Branch, Islamic Azad University (IAUPS), \\ Tehran 193956466, Iran.
}

Received 18 January 2016, revised 12 June 2016, accepted 15 July 2016.

\begin{abstract}
A simple, rapid, cost-effective and green high-performance liquid chromatographic assay for determination of capecitabine in human plasma using a $\mathrm{C}_{18}$ reversed-phase analytical column was developed and validated. The separation was conducted by means of a mobile phase composed of formic acid solution $(\mathrm{pH}=3)$ : ethanol (55:45) running at a flow-rate of $1.0 \mathrm{~mL} \mathrm{~min}{ }^{-1}$ with $\mathrm{UV}$ detection at $310 \mathrm{~nm}$. The column temperature was set at $50{ }^{\circ} \mathrm{C}$. Sample preparation involved protein precipitation by zinc sulfate-ethanol solution. This method is consistent with a high recovery of capecitabine in human plasma ranging from 95.98 to102.50\%.The calibration curves were linear over concentration range of $0.05-10.00 \mu \mathrm{g} \mathrm{mL} \mathrm{L}^{-1}\left(r^{2}>0.9999\right)$. Between- and within-day variability was less than $15 \%$ and the bias was within $\pm 15 \%$. This validated method was successfully applied to a pharmacokinetic study enrolling seven Iranian cancer patients after administration of a morning oral dose of $1500 \mathrm{mg}$.
\end{abstract}

KEYWORDS

Capecitabine, RP-HPLC method, ecofriendly, pharmacokinetics, human plasma.

\section{Introduction}

Colorectal and breast cancers, two of the most common types of neoplasms, are among the leading causes of death worldwide. These two diseases share some risk factors including smoking, exposure to environmental pollutants, physical inactivity, inadequate diet, excessive alcohol consumption and increased bodymass index from overweight to obese. ${ }^{1-2}$ Thus, living a healthier lifestyle could be one of the strategies to prevent cancer. Beyond this, medical therapies are an essential part of cancer treatment.

Capecitabine is an oral 5-fluorouracil (5-FU) pro-drug commonly given to patients suffering from colorectal and breast cancer. It is rapidly and completely absorbed through the gastrointestinal wall as an intact molecule. This drug is first metabolized in the liver into $5^{\prime}$-deoxy-5-fluorocytidine (5'-DFCR) by human carboxyesterase isoenzyme-2 (hCES2). The metabolite is then converted into 5'-deoxy-5-fluorouridine ( $5^{\prime}$-DFUR) by cytidine deaminase in tumor cells and in the liver. Finally, $5^{\prime}$-DFUR is metabolized by thymidine phosphorylase into the cytotoxic agent 5 -FU inside tumor cells. ${ }^{3-5}$ The metabolism pathway of capecitabine is shown in Fig. 1.

During the past years, the healthcare costs especially for the anti-cancer drugs have progressively increased worldwide, and this has made the governments and authorities to consider regulations on drug pricing. It is therefore essential that high-quality, low-cost drug formulations are made available. ${ }^{6}$ Conducting bioequivalence studies is of paramount importance to be sure about the efficacy and quality of such generic drugs. Regarding capecitabine, the Office of Generic Drugs (OGD) of the FDA

* To whom correspondence should be addressed. E-mail: afsharme@ sina.tums.ac.ir recommends a bioequivalence study to be carried out in cancer patients already receiving a stable dose $(1250 \mathrm{mg} / \mathrm{m} 2$ twice daily) of this medicine. In this respect, both the FDA and the European Medicines Agency (EMA) bioequivalence guidelines ${ }^{7-9}$ are on
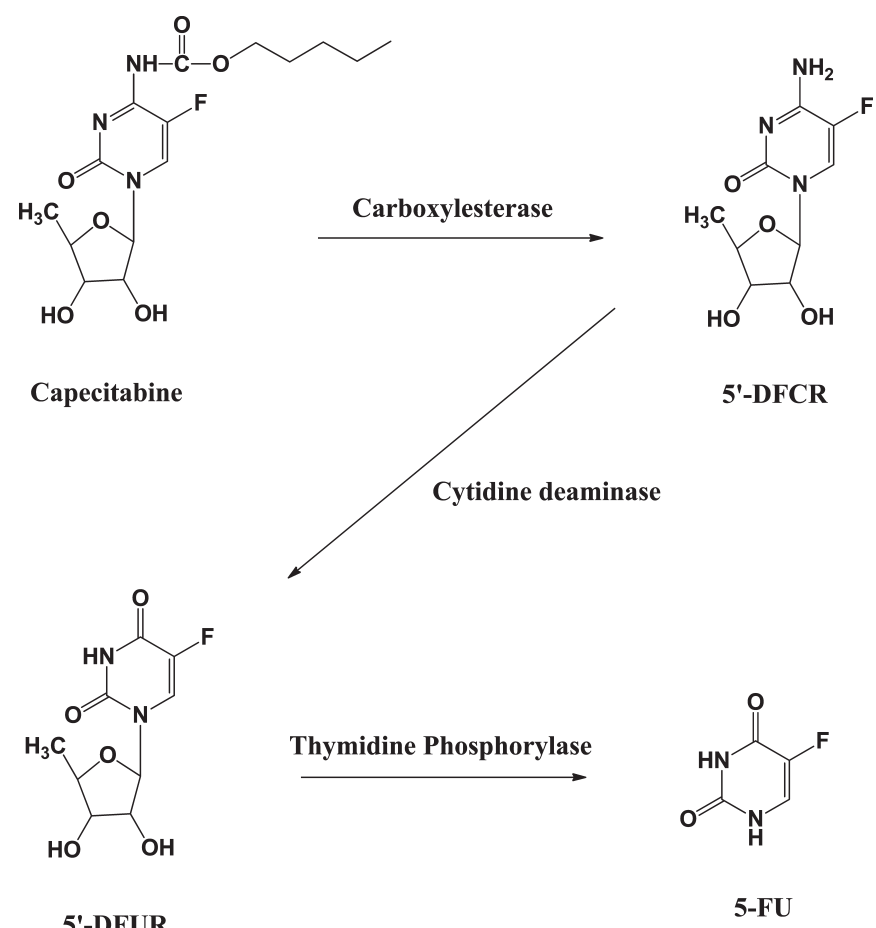

Figure 1 Metabolic pathway of capecitabine. 
the basis of comparison of capecitabine pharmacokinetic parameters obtained from the administration of the test and reference formulations. Therefore, simple chromatography methods for the bioanalysis of this drug should be available.

The most common methods to extract capecitabine from the plasma are liquid-liquid and solid-phase extraction, which are energy- and time-consuming sample-processing methods. ${ }^{10-13}$ Moreover, in the most previously reported procedures, mass spectrometry has been used as a detection system. ${ }^{13-18}$ These methods are highly specific and sensitive but involve expensive equipments which are not generally available in a clinical setting. The most important drawback of all previously published methods is the use of toxic and non-ecofriendly solvents such as acetonitrile in liquid chromatography or sample preparation steps. Because of inherent toxicity of such solvents, ${ }^{19}$ safe detoxification of the waste is essential, which may lead to high to very high disposal costs. Otherwise, the analysis itself may be a source of environmental pollution. Therefore, it is reasonable to apply greener and more sustainable approaches in bioanalysis to avoid polluting the environment through such experiments.

This paper describes a rapid and sensitive and greener HPLC-UV method for analyzing capecitabine using direct protein precipitation. The method was successfully applied to assess pharmacokinetics of capecitabine following administration of a $1500 \mathrm{mg}$ morning dose in seven cancer patients.

\section{Experimental}

\subsection{Materials, Reagents and Chemicals}

Qualified capecitabine standard $(99.0 \%)$ was kindly provided by Osveh Pharmaceuticals (Tehran, Iran). Absolute ethanol, gradient grade methanol and analytical grade reagents were purchased from Merck (Darmstadt, Germany). HPLC grade water was obtained through a Milli-Q system (Millipore, Milford, MA, USA) and was used to prepare all the solutions.

\subsection{Preparation of Standard Solutions}

Standard stock solution $\left(1.0 \mathrm{mg} \mathrm{mL}^{-1}\right)$ of capecitabine was prepared in methanol and stored in refrigerator at $4 \pm 2{ }^{\circ} \mathrm{C}$. This solution was found to be stable for at least one month at this temperature. Standard solutions were prepared daily from this solution by dilution with HPLC grade water.

\subsection{Sample Preparation}

An aliquot of $400 \mu \mathrm{L}$ of human plasma was mixed with $300 \mu \mathrm{L}$ of absolute ethanol and $30 \mu \mathrm{L}$ of zinc sulfate solution (17\%) to precipitate the plasma proteins. The mixture was vortexed for 1 min and centrifuged at $15800 \times g$ in a 5415C Eppendorf centrifuge for $10 \mathrm{~min}$. The clear supernatant was transferred into a clean tube and a volume of $100 \mu \mathrm{L}$ was injected into HPLC column.

\subsection{Apparatus and Chromatographic Conditions}

The HPLC method was carried out on a Younglin (Hogye, South Korea), which was equipped with YL9104 Vacuum degasser, YL9110 Quaternary pump, YL9131 Column compartment, and YL9120 UV/VIS detector. The peak areas were integrated automatically by computer using an Autochro-3000 software program. A $100 \mu \mathrm{l}$ volume of sample was introduced into a Rheodyne model 7725i injector. The elution was carried out on a C18 column $(150 \mathrm{~mm} \times 4.6 \mathrm{~mm}, 5 \mu \mathrm{m}$ particle size $)$ from Teknokroma (Barcelona, Spain) protected with a C18 guard column Teknokroma (Barcelona, Spain). All analyses were per- formed at the column temperature of $50 \pm 1{ }^{\circ} \mathrm{C}$ under isocratic conditions with a mobile phase consisting of a solution of formic acid in water $(\mathrm{pH}=3)$ : ethanol $(55: 45)$ and a flow-rate of

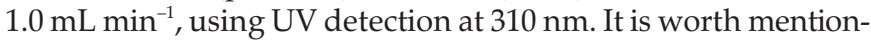
ing that the analysts were trained to manage the aqueous solutions containing this compound and also the potentially contaminated surfaces according to the previously published procedure. ${ }^{20}$

\subsection{Data Evaluation and Method Validation}

Method validation was carried out according to the FDA Guidance for Industry Bioanalytical Method Validation. ${ }^{21}$ Data were evaluated using peak areas.

\subsubsection{Preparation of the Calibration Standards and Quality Control Samples}

In order to prepare quality control samples (QC) and calibration standards, firstly $100 \mu \mathrm{L}$ of stock solution was vortex mixed with $900 \mu \mathrm{L}$ of HPLC grade water. Then $100 \mu \mathrm{L}$ of the prepared solution $\left(100 \mu \mathrm{g} \mathrm{mL}^{-1}\right)$ was added into $900 \mu \mathrm{L}$ of drug-free human plasma obtained from Iranian Blood Transfusion Organization (Tehran, Iran) and stepwise the final concentrations of $0.05,0.25$, $0.50,2.50,5.00$, and $10.00 \mu \mathrm{g} \mathrm{mL}^{-1}$ for the calibration standards and $0,0.05,0.13,1.00,7.50 \mu \mathrm{g} \mathrm{mL}^{-1}$ for QC samples were prepared. The resulting mixtures were subjected to the sample pretreatment described in sample preparation section and injected into HPLC.

Five sets of such calibrators were prepared on five subsequent days. Each calibrator was injected in triplicate. Calibration data were acquired by plotting the peak area of capecitabine against the concentrations of the calibration standards, followed by a linear regression analysis.

\subsubsection{Precision, Accuracy, Limit of Quantification and Recovery}

Five replicate QC samples were injected between and within day. The assay precision was expressed as a percentage of RSD. Accuracy was calculated as the deviation of the mean from nominal concentration. The LOQ was defined as the lowest concentration of capecitabine at which the percentage deviation from the nominal concentration (accuracy) and the RSD were within $\pm 20 \%$ and less than $20 \%$, respectively, considering at least ten times the response compared to that of the blank response. The recovery of capecitabine was determined by comparing the peak area of the processed QC samples with those obtained after the injection of the same amount of drug dissolved in HPLC grade water at different concentration levels.

\subsubsection{Specificity and Selectivity}

Control human plasma, obtained from 13 patients was assessed by aforementioned procedure and compared with respective spiked plasma samples to evaluate the selectivity of the method.

\subsection{Application of the Method}

Nine cancer patients (five males and four females) with histologically proven cancer who were treated with capecitabine (1500 mg twice daily) alone or together with other commonly used medicines in breast or colon cancer like cetuximab, dexamethasone, oxaliplatin were included in this study. The ages of patients were in the range of 54-79 years. Patients with a history of cardiovascular, renal or hepatic disorder were excluded from this study. The patients received $1500 \mathrm{mg}$ capecitabine (Xeloda ${ }^{\circledR}$; Hoffmann-La Roche Ltd., Basle, Switzerland) as a single oral dose after an overnight fast. The intake of food was delayed for $3 \mathrm{~h}$ post oral dose. Peripheral venous blood samples ( $5 \mathrm{~mL}$ each) were collected into heparinized tubes from each patient imme- 
diately before and from 0.5 to $5 \mathrm{~h}$ after the morning dose. The blood samples were centrifuged immediately at $3000 \times g$ for $10 \mathrm{~min}$ and plasma samples were stored at $-20^{\circ} \mathrm{C}$ until analysis.

This study was approved by Azad University of Pharmaceutical Sciences ethics committee (Reference number: 17697) and informed consents were obtained from each individual patient.

\subsection{Calculation of the Pharmacokinetic Parameters}

Plasma concentration-time curves of capecitabine were evaluated using non-compartmental analysis. The values of maximum plasma concentration $\left(C_{\max }\right)$ and the time to $C_{\max }\left(T_{\max }\right)$ were directly obtained from experimental observations. The elimination rate constant $\left(k_{\mathrm{e}}\right)$ was obtained from a log-linear regression analysis of the plasma concentration-time curves in the terminal phase. The terminal half-life $\left(t_{1 / 2}\right)$ was calculated from $k_{\mathrm{e}}$. The area under the concentration-time curve from $0 \mathrm{~h}$ to the last quantifiable concentration $\left(\mathrm{AUC}_{0-t}\right)$ was calculated by the linear trapezoidal method. The area from the last measured concentration to infinity $\left(\mathrm{AUC}_{\mathrm{t}-\infty}\right)$ was calculated by dividing the last measurable plasma concentration by the $k_{\mathrm{e}}$. The clearance (CL) was calculated as fDose/AUC ${ }_{0-\infty}$. Since the bioavailability of capecitabine was reported to be $100 \%,{ }^{4} \mathrm{f}$ was considered to be equal to 1 .

The non-compartmental model analysis was performed on Microsoft Excel software version 2010. Data are expressed as mean \pm standard deviation $(\bar{X} \pm$ S.D. $)$.

\section{Results and Discussion}

\subsection{Selectivity, Specificity and Chromatography}

The HPLC procedure was optimized with a view to develop a greener bioanalytical method. Green analytical techniques aim to minimize or eliminate the hazardous waste associated with analytical methods. In this context, it is reasonable to use ethanol-water as the mobile phase of choice for many RP-HPLC applications. ${ }^{22}$ Therefore, ethanol was chosen as the organic modifier and different ratios of the ethanol and water were examined to obtain a symmetric peak of capecitabine with proper capacity factor. The best condition regarding retention time was achieved using a mobile phase consisting of ethanol:water $(45: 55, \mathrm{v} / \mathrm{v})$, but the column pressure was too elevated at the flow rate of $1.0 \mathrm{~mL} \mathrm{~min}{ }^{-1}$. Therefore, the temperature was increased to $50{ }^{\circ} \mathrm{C}$. Even so, the peak shape was not optimal under the aforementioned chromatographic condition. To solve this problem, water was replaced by a solution of formic acid in water $(\mathrm{pH}=3)$. This change resulted in rather symmetric peaks with tailing factor of $1.31 \pm 0.83$ and theoretical plates of more than 2000. Figure 2 shows the chromatograms obtained from the method development.

According to these preliminary results, the detection wavelength of $310 \mathrm{~nm}$, flow rate of $1.0 \mathrm{~mL} \mathrm{~min}^{-1}$ and the mobile phase of formic acid solution $(\mathrm{pH}=3)$ :ethanol $(55: 45, \mathrm{v} / \mathrm{v})$, were finalized. Under such chromatographic condition, capecitabine peak appeared at about $3.81 \pm 0.06 \mathrm{~min}(n=10)$ and no endogenous plasma component was eluted at this retention time, as evaluated by chromatograms of blank human plasma obtained from 13 patients and plasma spiked with capecitabine. A representative chromatogram from a plasma sample obtained $0.5 \mathrm{~h}$ after oral administration of capecitabine-based treatment from a patient, the blank plasma of the same patient and a chromatogram collected from human blank plasma spiked with

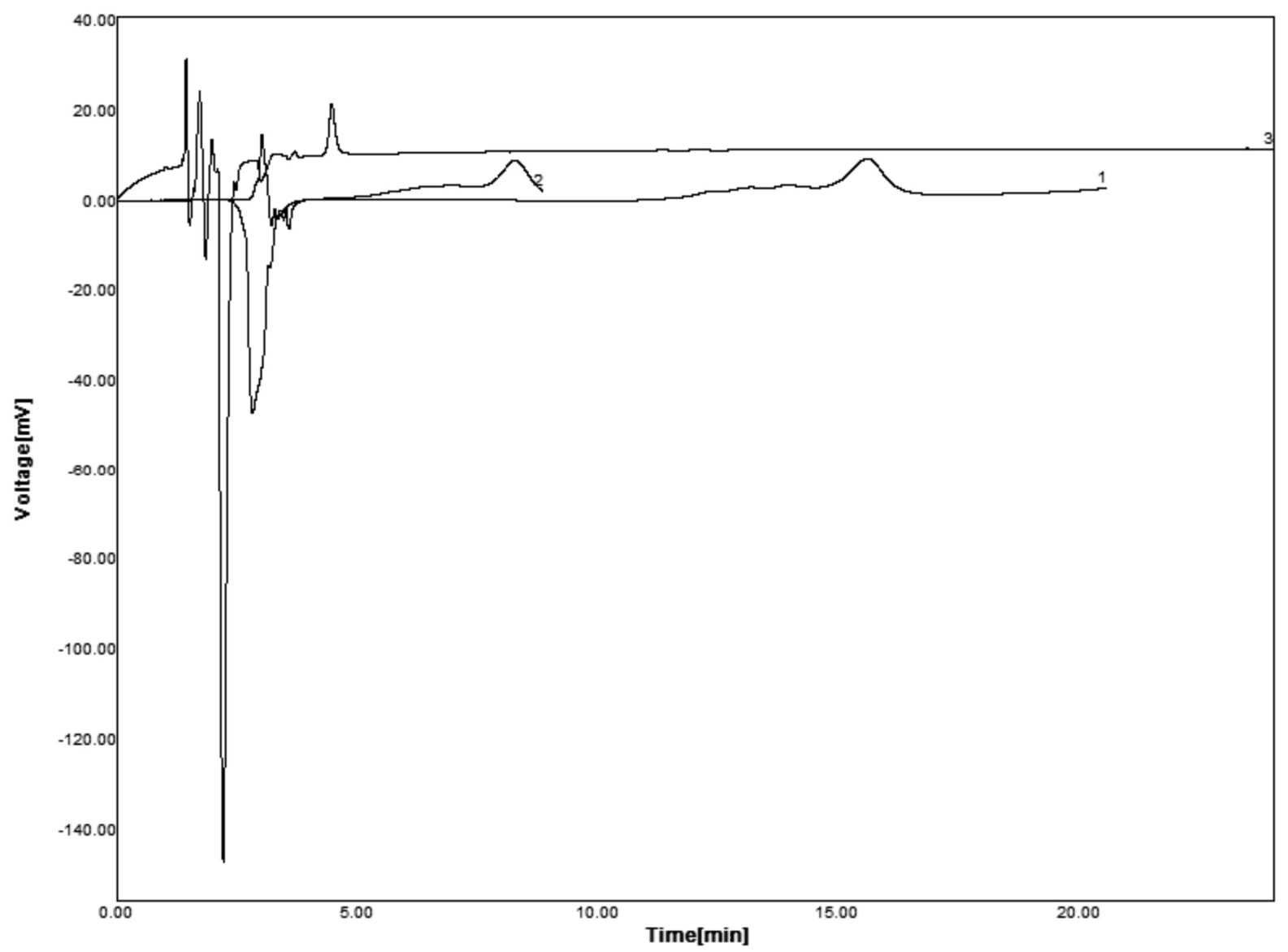

Figure 2 Chromatograms (1) and (2) were obtained from the injection of a standard solution of capecitabine in water using mobile phases consisting of $20 \%$ and $40 \%$ ethanol in HPLC grade water, respectively. Chromatogram 3 is the result of injection of the same standard solution using a mobile phase consisting of a solution of formic acid in water $(\mathrm{pH}=3)$ : ethanol $(56: 44)$. The column temperature was $50^{\circ} \mathrm{C} \pm 1{ }^{\circ} \mathrm{C}$ for the last chromatogram. 
$2.50 \mu \mathrm{g} \mathrm{mL}^{-1}$ capecitabine are shown in Fig. 3. It is worth mentioning that the major capecitabine metabolites like 5-flourouracil, 5'-DFCR and 5'-DFUR show their maximum absorbance at around 266 and $205 \mathrm{~nm}$ and do not have UV absorbance at $310 \mathrm{~nm} \cdot{ }^{9-10}$ Therefore, they do not interfere with capecitabine assay at the chosen wavelength. Also, the co-administered drugs did not give any interfering peaks. No change in column efficacy and back pressure was observed over the entire study time.

\subsection{Sample Preparation}

The recovery of capecitabine was assessed using greener analytical solvents, ethanol and methanol. Although addition of three volumes ethanol to one volume of plasma produced complete precipitation of the plasma proteins, it was not satisfactory because of the higher LOQs. Lower LOQs and higher recoveries were achieved by adding zinc sulfate to ethanol. Interestingly, ethanol yielded sharper peaks and as a result lower LOQs compared to methanol. As shown in Table 1, the recoveries achieved in this study are in the range of $95.98-102.50 \%$.

\subsection{Method Validation}

Assay linearity was evaluated over the concentration range of $0.05-10.00 \mu \mathrm{g} \mathrm{mL}^{-1}$, constructing six-point calibration curves. The equations for means of five standard curves is $y=(93.25 x \pm$ $1.12)+(-2.10 \pm 0.26),\left(r^{2}=0.9999\right)$. Samples having concentration more than $10.00 \mu \mathrm{g} \mathrm{mL}^{-1}$ were first diluted with drug-free plasma. LOQ, as previously defined, was $0.05 \mu \mathrm{g} \mathrm{mL}{ }^{-1}$, considering the mean accuracy value of $98.69 \%$ and RSD value lower than $6.92 \%$, which seems to be proper to perform a bioequivalence and pharmacokinetic study. ${ }^{6,24-27}$ This LOQ is lower than those of previously reported procedures ${ }^{12,23}$ and comparable to that obtained using a HPLC-MS/MS method. ${ }^{16}$ RSD values for between- and within-day determinations were found to be in the range of 5.58-6.92\% and 1.90-6.66 \% , respectively (Table 1). These values document the high precision of the assay. They fulfill the validation criteria of an analytical method designed for pharmacokinetic and drug metabolism studies for which RSD values of less than $15 \%$ are acceptable. Moreover, the accuracy was found to be in the range of 92.68-102.67\% (Table 1), that is acceptable for testing drug content in biological samples.

The stability of processed and non-processed quality control samples was assessed after four freeze-thaw cycles and after being kept for at least $5 \mathrm{~h}$ at ambient temperature $(n=3)$, respectively. The obtained accuracy and RSD values were in the range of $85-115 \%$ and less than $15 \%$, respectively. These data confirm the stability of these samples.

\subsection{Application of the Method}

The proposed method was applied to determine the pharmacokinetic parameters of nine patients after receiving a $1500 \mathrm{mg}$ (three $500 \mathrm{mg}$ tablets) dose of capecitabine. In two out of nine patients, double peak phenomenon was observed in
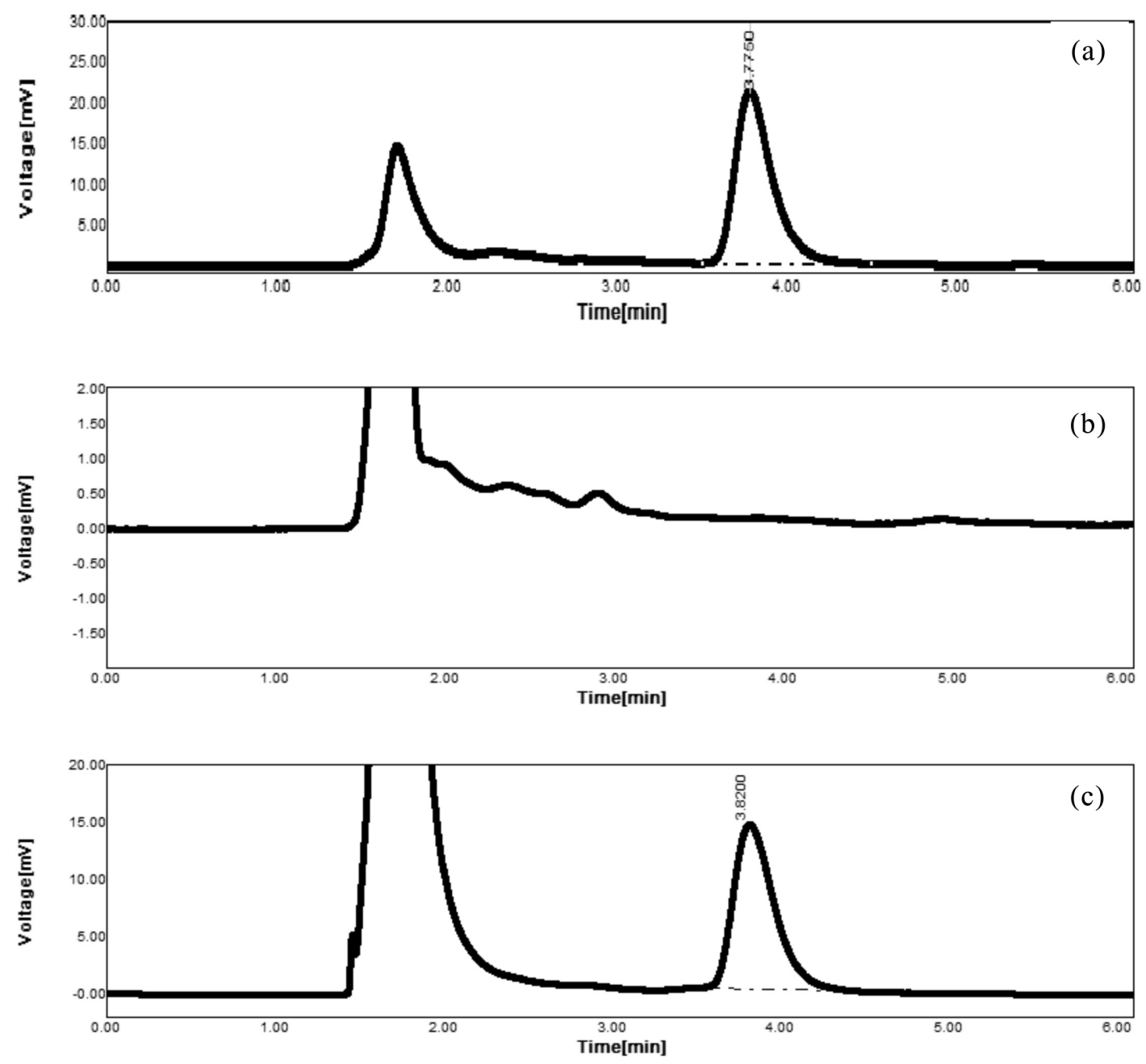

Figure 3 Chromatograms of (a) plasma of a patient $0.5 \mathrm{~h}$ after oral administration of a $1500 \mathrm{mg}$ dose of capecitabine, (b) blank plasma of the same patient and (c) plasma spiked with $2.5 \mu \mathrm{g} \mathrm{mL}^{-1}$ capecitabine. 
Table 1 Between- and within-day variability, accuracy and recovery for determination of capecitabine.

\begin{tabular}{|c|c|c|c|c|c|c|}
\hline \multirow[t]{2}{*}{$\begin{array}{l}\text { Concentration } \\
/ \mu \mathrm{g} \mathrm{mL}^{-1}\end{array}$} & \multicolumn{2}{|c|}{$\begin{array}{l}\text { Between-day variability } \\
\qquad / n=5\end{array}$} & \multicolumn{2}{|c|}{$\begin{array}{l}\text { Within-day variability } \\
\qquad / n=5\end{array}$} & \multicolumn{2}{|c|}{$\begin{array}{l}\text { Recovery } \\
\quad / n=5\end{array}$} \\
\hline & $\mathrm{RSD} / \%$ & Accuracy $/ \%$ & $\mathrm{RSD} / \%$ & Accuracy $/ \%$ & $\overline{\mathrm{RSD} / \%}$ & $\%$ \\
\hline 0.05 & 6.92 & 94.70 & 3.13 & 102.67 & 4.25 & 100.27 \\
\hline 0.13 & 6.35 & 92.68 & 6.66 & 100.49 & 2.67 & 102.50 \\
\hline 1.00 & 5.65 & 99.18 & 3.06 & 95.38 & 1.36 & 95.98 \\
\hline 7.50 & 5.58 & 99.28 & 1.90 & 99.26 & 4.20 & 99.53 \\
\hline
\end{tabular}

plasma concentration-time profiles, which could be due to disintegration of the tablets in gastrointestinal tract. The tablet that disintegrates slower may have delayed gastric emptying, resulting in a second absorption peak. Because there was not enough information in the terminal phase to calculate $k_{\mathrm{e}}$, these two patients were excluded from the pharmacokinetic study. The $C_{\max }, T_{\max }, \mathrm{AUC}_{0-\infty}, t_{1 / 2}$ and $C L$ in the remaining seven patients were calculated to be $6.29 \pm 5.06 \mu \mathrm{g} \mathrm{mL}^{-1}, 0.71 \pm 0.27 \mathrm{~h}, 5.78 \pm$ $3.22 \mu \mathrm{g} \mathrm{h} \mathrm{mL}^{-1}, 0.53 \pm 0.15 \mathrm{~h}$ and $345.31 \pm 199.67 \mathrm{~L} \mathrm{~h}^{-1}$, respectively. The typical plot of the capecitabine concentrations as a function of time in a patient is depicted in Fig. 4. The results of the main pharmacokinetic parameters are summarized in Table 2. Considering the fact that, capecitabine exhibits linear increases in $C_{\max }$ and $\mathrm{AUC}_{0-\infty}$ when dosage increases, ${ }^{4}$ it is possible to normalize the previously reported pharmacokinetic data to a dose of $1500 \mathrm{mg}$. Therefore, it can be concluded that the pharmacokinetic parameters obtained in this study were similar to the published pharmacokinetic studies ${ }^{26,28}$ and it seems that there are no racial differences in capecitabine pharmacokinetics.

\section{Conclusion}

In this study a simple, economical and sensitive method for determination of capecitabine in human plasma was developed and fully validated on the basis of specificity, linearity, sensitivity, precision and accuracy, as well as recovery and stability. The method provided satisfactory sensitivity using a simple and fast protein precipitation procedure with total sample preparation time of about $15 \mathrm{~min}$ and did not involve any drying or reconstitution steps to achieve the desired sensitivity. This is suitable for the analysis of large numbers of biological samples, which normally exist in a bioequivalence or pharmacokinetic study and is in accordance with one of the principles of green analytical chemistry, which emphasizes on designing for energy efficiency. ${ }^{29}$ The principal advantage of the method is the use of available environmentally friendly solvents for LC analyzing and sample preparation to follow the first principle of green chemistry which emphasizes on the waste prevention instead of remediation. ${ }^{19}$ To the best of our knowledge this is the first method which has been introduced as 'green'. The assay was

Table 2 Individual pharmacokinetic data obtained from seven patients following oral administration of $1500 \mathrm{mg}$ of capecitabine.

\begin{tabular}{|c|c|c|c|c|c|}
\hline Patient & $T_{\max }$ & $\begin{array}{c}C_{\max } \\
/ \mu \mathrm{g} \mathrm{mL}^{-1}\end{array}$ & $\begin{array}{l}\mathrm{AUC}_{(0-\infty)} \\
/ \mu \mathrm{g} \mathrm{h} \mathrm{mL}^{-1}\end{array}$ & $\begin{array}{l}t_{1 / 2} \\
/ \mathrm{h}\end{array}$ & $\begin{array}{c}\mathrm{CL} \\
/ \mathrm{L} \mathrm{h}^{-1}\end{array}$ \\
\hline 1 & 0.50 & 16.19 & 11.28 & 0.42 & 132.98 \\
\hline 2 & 0.50 & 2.99 & 2.80 & 0.47 & 535.71 \\
\hline 3 & 1.00 & 1.78 & 2.21 & 0.69 & 678.73 \\
\hline 4 & 1.00 & 2.05 & 3.91 & 0.33 & 383.63 \\
\hline 5 & 0.50 & 6.39 & 5.44 & 0.53 & 275.74 \\
\hline 6 & 0.50 & 8.81 & 8.27 & 0.54 & 181.38 \\
\hline 7 & 1.00 & 5.85 & 6.55 & 0.75 & 229.01 \\
\hline Mean & 0.71 & 6.29 & 5.78 & 0.53 & 345.31 \\
\hline S.D. & 0.27 & 5.06 & 3.22 & 0.15 & 199.67 \\
\hline
\end{tabular}

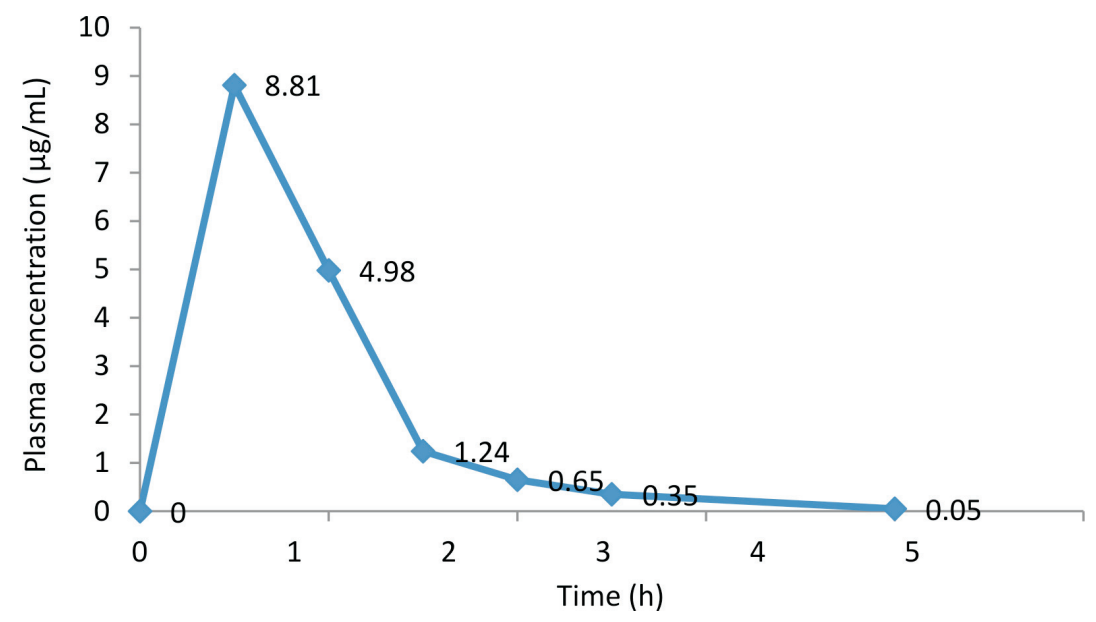

Figure 4 Plasma concentration-time profile of capecitabine after administration of a $1500 \mathrm{mg}$ morning dose to a cancer patient. 
successfully used in pharmacokinetic study of capecitabine after administration of a single oral dose of $1500 \mathrm{mg}$ in seven Iranian cancer patients.

\section{Acknowledgements}

This paper is a part of Pharm.D. thesis of Sepideh Hassanlou. The authors extend their appreciation to Osveh Pharmaceutical Co. for kind donation of capecitabine standard. We also thank the patients for their participation.

The authors declared no conflict of interest.

\section{References}

1 H. Maalmi, J.M. Ordóñez-Mena, B. Schöttker and H. Brenner, Serum 25-hydroxyvitamin D levels and survival in colorectal and breast cancer patients: systematic review and meta-analysis of prospective cohort studies, Eur. J. Cancer., 2014, 50, 1510-1521. DOI 10.1016/j.ejca.2014.02.006

2 A.A. Ghazarian, N.I. Simonds, K. Bennett, C.B. Pimentel, G.L. Ellison, E.M. Gillanders, S.D. Schully and L.E. Mechanic, A review of NCI's extramural grant portfolio: identifying opportunities for future research in genes and environment in cancer, Cancer Epidemiol. Biomarkers. Prev, 2013, 22, 501-507. DOI: 10.1158/1055-9965.EPI-13-0156

3 V. Schreiber, M. Kitzmueller, M. Poxhofer, S. Gintersdorfer, C. Neudorfer, M. Lichtneckert, C. Dittrich and M. Czejka, Impact of co-administered drugs on drug monitoring of capecitabine in patients with advanced colorectal cancer, Anticancer Res., 2014, 34, 3371-3376.

4 C.M. Walko and C. Lindley, Capecitabine: a review, Clin. Ther, 2005, 27, 23-44. DOI: 10.1016/j.clinthera.2005.01.005

5 S. Urien, K. Rezaí, F. Lokiec, Pharmacokinetic modelling of 5-FU production from capecitabine - a population study in 40 adult patients with metastatic cancer, J. Pharmacokinet. Pharmacodyn., 2005, 32(5-6), 817-833. DOI: 10.1007/s10928-005-0018-2 ‘This reference was added based on Plagiarism report

6 S. Chachad, S. Purandare, G. Malhotra and R. Naidu, Comparison of pharmacokinetics and safety profiles of two capecitabine tablet formulations in patients with colon, colorectal or breast cancer, Cancer Chemother. Pharmaco., 2013, 71, 287-292. DOI: 10.1007/s00280-0122007-7

7 C. Gadiko, S. K. Tippabhotla, S. Thota, R. Battula, M. Nakkawar, S. Yergude, S. Md. Khan, R. Cheerla, M.R. Betha and V. Vobalaboina, Comparative bioavailability study of capecitabine tablets of $500 \mathrm{mg}$ in metastatic breast cancer and colorectal cancer patients under fed condition, Clin. Res. Regulat. Affairs, 2012, 29, 72-76. DOI: 10.3109/10601333.2012.752496

8 CHMP/PKWP/EMA/423732/2013, 24 October 2013

9 FDA Draft Guidance on Capecitabine, Recommended Jul 2008; Revised Aug 2010; Sep 2012

10 L. Zufía, A. Aldaz and J. Giráldez, Simple determination of capecitabine and its metabolites by liquid chromatography with ultraviolet detection in a single injection, J. Chromatogr. B. Analyt Technol Biomed Life Sci., 2004, 809, 51-58. DOI: 10.1016/j.jchromb.2004.06.004

11 E. Piórkowska, M. Kaza, J. Fitatiuk, I. Szlaska, T. Pawiñski and P.J. Rudzki, Rapid and simplified HPLC-UV method with on-line wavelengths switching for determination of capecitabine in human plasma, Pharmazie, 2014, 69, 500-505. DOI: 10.1691/ph.2014.3223

12 A. Farkouh, D. Ettlinger, J. Schueller, A. Georgopoulos, W. Scheithauer and M. Czejka, A rapid and simple HPLC assay for quantification of capecitabine for drug monitoring purposes, Anticancer Res., 2010, 30, 5207-5211.

13 D. Montange, M. Bérard, M. Demarchi, P. Muret, S. Piédoux, J.P. Kantelip and B. Royer, An APCI LC-MS/MS method for routine determination of capecitabine and its metabolites in human plasma, J. Mass. Spectrom., 2010, 45, 670-677. DOI: 10.1002/jms.1759

14 L.D. Vainchtein, H. Rosing, J.H. Schellens and J.H. Beijnen, A new validated HPLC-MS/MS method for the simultaneous determina- tion of the anti-cancer agent capecitabine and its metabolites: 5 -deoxy-5-fluorocytidine, 5'-deoxy-5-fluorouridine, 5-fluorouracil and 5-fluorodihydrouracil, in human plasma, Biomed. Chromatogr., 2010, 24, 374-386. DOI: 10.1002/bmc.1302

15 H. Licea-Perez, S. Wang and C. Bowen, Development of a sensitive and selective LC-MS/MS method for the determination of alphafluoro-beta-alanine, 5-fluorouracil and capecitabine in human plasma, J. Chromatogr. B. Analyt. Technol. Biomed. Life Sci., 2009, 877, 1040-1046. DOI: 10.1016/j.jchromb.2009.02.025

16 M.J. Deenen, H. Rosing, M.J. Hillebrand, J.H. Schellens and J.H. Beijnen, Quantitative determination of capecitabine and its six metabolites in human plasma using liquid chromatography coupled to electrospray tandem mass spectrometry, J. Chromatogr. B. Analyt. Technol. Biomed. Life Sci., 2013, 30-40. DOI: 10.1016/ j.jchromb.2012.11.033

17 S.M. Guichard, I. Mayer and D.I. Jodrell, Simultaneous determination of capecitabine and its metabolites by HPLC and mass spectrometry for preclinical and clinical studies, J. Chromatogr. B. Analyt. Technol. Biomed. Life Sci., 2005, 826, 232-237. DOI: 10.1016/j.jchromb.2005.09.010

18 P. Deng, C. Ji, X. Dai, D. Zhong, L. Ding and X. Chen, Simultaneous determination of capecitabine and its three nucleoside metabolites in human plasma by high performance liquid chromatographytandem mass spectrometry, J. Chromatogr. B Analyt. Technol. Biomed. Life Sci., 2015, 989, 71-79. DOI: 10.1016/j.jchromb.2015.03.002

19 R.A. Sheldon, Fundamentals of green chemistry: efficiency in reaction design, Chem. Soc. Rev., 2012, 41, 1437-1451. DOI: 10.1039/c1cs15219j

20 A.A. Shahrasbi, M. Afshar, F. Shokraneh, F. Monji, M. Noroozi, M. Ebrahimi-Khojin, S.F. Madani, M. Ahadi-Barzoki, M. Rajabi, EXCLI. J. 2014, 13, 491-501. eCollection 2014

21 Guidance for industry, bioanalytical method validation. US Department of Health and Human Services, Food and Drug Administration, May 2001.

22 J. Płotka, M. Tobiszewski, A.M. Sulej, M. Kupska, T. Górecki and J. Namiśnik, Green chromatography, J. Chromatogr. A., 2013, 1307, 1-20. DOI: 10.1016/j.chroma.2013.07.099

23 S. Jayaseelan, S.k. Bajivali, U. Ramesh, V. Sekar and P. Perumal, Bioanalytical method development and validation of capecitabine by RP-HPLC method, Int. J. Chem. Tech. Res., 2010, 2, 2086-2090.

24 P. Roxburgh, G.R Lumsden, J. Paul, S. Harden, L. Sweeting, A. James, A. Crellin, R. Morrison, T.R. Evans and A.C. McDonald, A phase I and pharmacokinetic study of capecitabine in combination with radiotherapy in patients with localised inoperable pancreatic cancer, Cancer Chemother. Pharmacol., 2014, 74, 131-139. DOI: 10.1007/s00280014-2470-4

25 A. Farkouh, W. Scheithauer, P. Buchner, A. Georgopoulos, J. Schueller, B. Gruenberger and M. Czejka, Clinical pharmacokinetics of capecitabine and its metabolites in combination with the monoclonal antibody bevacizumab, Anticancer Res., 2014, 34, 3669-3673.

26 B. Reigner, J. Verweij, L. Dirix, J. Cassidy, C. Twelves, D. Allman, E. Weidekamm, B. Roos, L. Banken, M. Utoh and B. Osterwalder, Effect of food on the pharmacokinetics of capecitabine and its metabolites following oral administration in cancer patients, Clin. Cancer Res., 1998, 4, 941-948.

27 X. Pivot, E. Chamorey, E.N. Guardiola, A. Magné, J. Thyss Otto, B. Giroux, Z. Mouri, M. Schneider and G. Milano, Phase I and pharmacokinetic study of the association of capecitabine-cisplatin in head and neck cancer patients, Ann. Oncol., 2003, 14, 1578-1586. DOI: 10.1093/annonc/mdg410

28 B. Reigner, T. Watanabe, J. Schüller, H. Lucraft, Y. Sasaki, J. Bridgewater, T. Saeki, J. McAleer, M. Kuranami, C. Poole, M. Kimura, J. Monkhouse, C. Yorulmaz, E. Weidekamm and S. Grange, Pharmacokinetics of capecitabine (Xeloda) in Japanese and Caucasian patients with breast cancer, Cancer Chemother. Pharmacol., 2003, 52, 193-201. DOI: $10.1007 / \mathrm{s} 00280-003-0642-8$

29 C. Ghosh, Green bioanalysis: some innovative ideas towards green analytical techniques, Bioanalysis, 2012, 4, 1377-1391. DOI: 10.4155/bio.12.31 